\title{
Polarized Molecules: A new Option for Internal Storage-Cell Targets?
}

\author{
Ralf Engels* \\ Institut für Kernphysik, Forschungszentrum Jülich, \\ Wilhelm-Johnen-Str. 1, 52428 Jülich, Germany \\ E-mail: r.w.engelsefz-juelich.de
}

In the last decades different types of storage cells have been used to increase the target density of polarized internal targets in storage rings, fed with atoms from an atomic beam source. Most of these cells are optimized to avoid recombination of the polarized hydrogen or deuterium atoms into molecules and to preserve the nuclear polarization at a high level between 0.75 and 0.9 . Independently, groups at AmPS [1], IUCF [2], and HERMES [3] have shown that nucleons of recombined molecules can still be polarized. In a collaboration between the Petersburg Nuclear Physics Institute, the University of Cologne and the Forschungszentrum Jülich we have built a dedicated apparatus to measure the polarization of hydrogen(deuterium) atoms and molecules in cells of different surface materials for temperatures between 45 and $120 \mathrm{~K}$ and in magnetic fields up to $1 \mathrm{~T}$. In addition, the recombination probability of atoms and the amount of wall bounces of molecules inside the cells have been measured with good precision. First results and possible options for further use will be presented.

XVIth International Workshop in Polarized Sources, Targets, and Polarimetry, PSTP2015,

14-18 September 2015,

Bochum, Germany

${ }^{*}$ Speaker. 


\section{Introduction}

The use of polarized beams and/or targets is an important asset in nuclear and hadron physics experiments. Storage cells are frequently employed to increase the areal density of polarized hydrogen and deuterium gas targets over that achieved with jets of polarized atoms [4]. The cells are optimized to minimize depolarization of the atoms by wall collisions and by recombination to molecules, in order to maintain the high nuclear polarization of the cell-feeding beams from atomic beam sources (ABSs). Different designs of these storage cells, e.g. longer cells with smaller inner diameter or cells that can be closed after acceleration of the beam to shrink the inner diameter moreover, helped to increase the target density. A further increase of the target-gas density is achieved by cooling the cell wall to $100 \mathrm{~K}$ for hydrogen and $80 \mathrm{~K}$ for deuterium. Cooling to lower temperatures leads to depolarization of the atoms and an enhanced recombination [5, 6]. Experiments on the preservation of the nuclear polarization in the molecules after recombination of $\mathrm{D}$ [1] and $\mathrm{H}$ [2] on copper walls revealed values of about $50 \%$ of that of the atoms before recombination (requiring also a sufficient magnetic field along the storage cell). A slightly higher value was derived for $\mathrm{H}$ recombining in a storage cell coated by radiation-damaged Drifilm, a silicon-based polymer [3].

\section{The Apparatus}

Price and Haeberli supposed in 1993 [7] to measure the nuclear polarization of atoms and molecules in such storage cells by ionizing both particles with an electron beam in a strong magnetic field. Afterwards, the produced $\mathrm{H}_{2}^{+}$ions and protons should be accelerated into a Lamb-shift polarimeter [8]. Based on this idea a collaboration between the PNPI in St. Petersburg, the University of Cologne and the research center Jülich built a vacuum chamber with a superconducting solenoid at liquid helium temperatures (see Fig. 1). Inside this solenoid is a $40 \mathrm{~cm}$ long storage cell with $10 \mathrm{~mm}$ inner diameter made from fused quartz. The inner surface was covered with a thin gold film or other materials like liquid Fomblin. The cell can be cooled down to temperatures around $45 \mathrm{~K}$ via contact to the liquid helium tanks and can be heated up to $120 \mathrm{~K}$ without reasonable helium losses. To feed the cell with polarized hydrogen (deuterium) atoms an ABS [9] is mounted on top of the vacuum chamber. On the left side an electron gun produces an electron beam of 0.1 $1 \mathrm{~mA}$ at about $100 \mathrm{eV}$ beam energy which is focused through the storage cell. The cell itself can be set to a potential up to $+8 \mathrm{keV}$ to accelerate the ionized particles, protons and $\mathrm{H}_{2}^{+}$ions into the Lamb-shift polarimeter. During the measurements it was obvious that beside the polarization of the protons even the polarization of the $\mathrm{H}_{2}^{+}$ions and, therefore, of the molecules can be determined with the LSP directly [10]. In this case, one has to be aware that the Wien filter, depending on the magnetic field inside, will rotate the polarization of the protons, e.g. for $180^{\circ}$ for a change of sign. The direction of the polarization of the $\mathrm{H}_{2}^{+}$ions is dominated by the magnetic moment of the residual electron, which is much larger than that of the proton. The magnetic moment of the protons is now orientated by the direction of the electron spin. Therefore, the polarization of the $\mathrm{H}_{2}^{+}$ ions will follow adiabatically the direction of the magnetic field inside and will be perpendicular to the beam direction behind the Wien filter. Afterwards, both types of ions will reach the Cs cell where metastable hydrogen (deuterium) atoms are produced by charge exchange with the Cesium. During this process the nuclear polarization is conserved until a sufficient magnetic field (about 50 


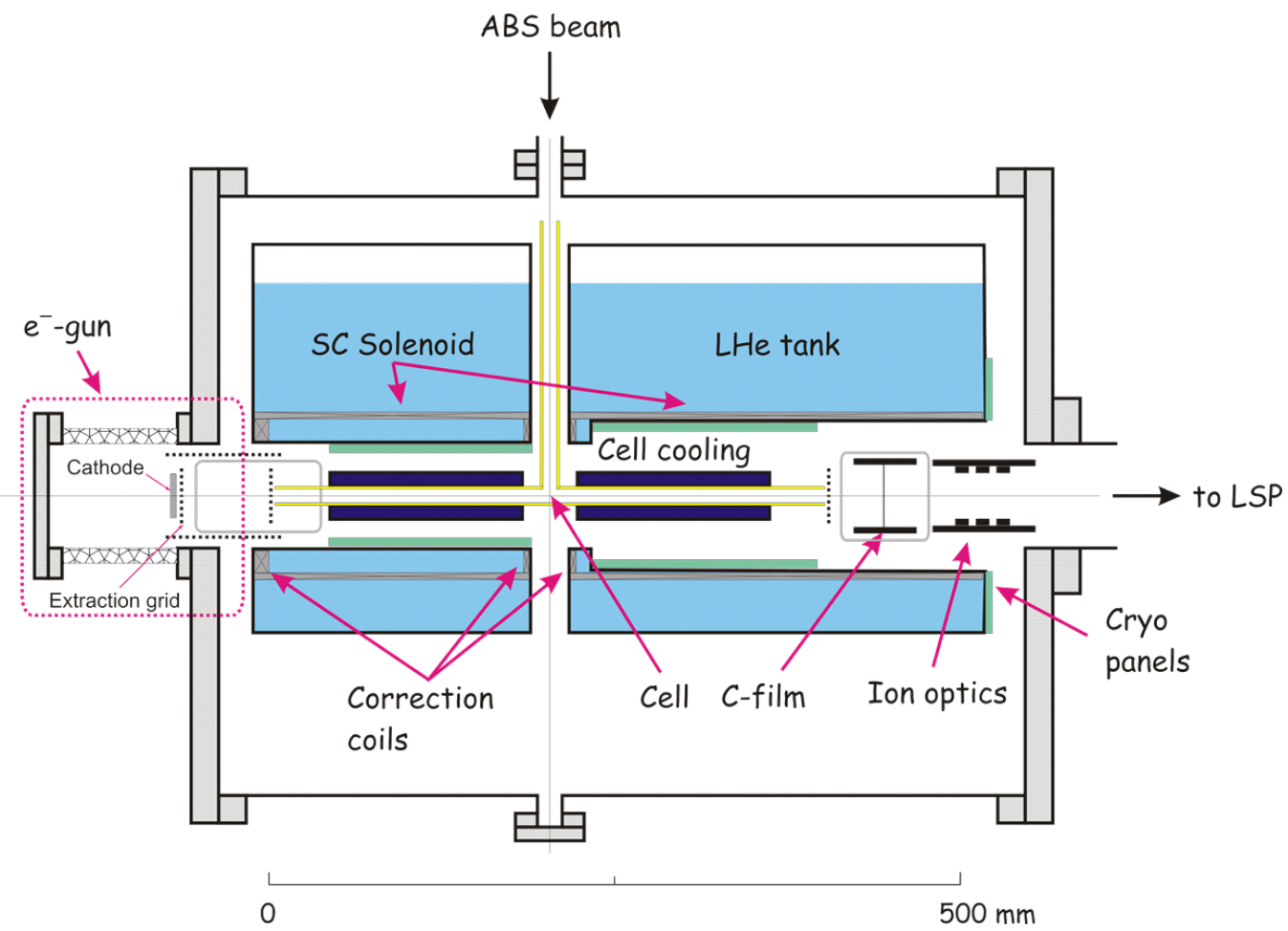

Figure 1: The general layout of the interaction chamber.

mT) is induced inside. Again, the polarization of the $\mathrm{H}_{2}^{+}$ions will follow this longitudinal field immediately and, therefore, will be parallel to the beam direction. Thus, the magnetic field of the Wien filter does not influence the polarization of the $\mathrm{H}_{2}^{+}$-ions, but it rotates the polarization of the protons (deuterons). The only difference in both processes is the cross section which is about 35 times smaller for the production of metastable atoms from the $\mathrm{H}_{2}^{+}$ions as compared to the protons. In the spinfilter the different hyperfine states are separated and the occupation numbers can be deduced when the residual metastable atoms are quenched into the ground state. The amount of produced Lyman- $\alpha$ photons depends directly on the amount of protons $/ H_{2}^{+}$-ions with the corresponding nuclear spin.

Another important detail is the extraction of the $\mathrm{H}_{2}^{+}$ions from the storage cell. Due to the pressure of $10^{-4}$ mbar inside the storage cell the average free path length of the $\mathrm{H}_{2}^{+}$ions is shorter than the length of the cell itself. Therefore, $H_{2}^{+}$ions produced in the center of the cell have a smaller chance to be extracted than the one from the end of the cell.

\section{First Results}

Because of the interaction of the nuclear spins $I$ with the rotational magnetic moment of the molecule $J$ some amount of polarization is lost, when the direction of the rotation axis is randomized during wall collisions. This effect can be decreased, if an external magnetic field is used to decouple both magnetic moments. Due to ref. [2] the polarization $P_{m}$ of the molecules after $n$ wall in a magnetic field $B$ is

$$
P_{m}(n, B)=P_{m_{0}} e^{-n\left(B_{c} / B\right)^{2}}
$$


$P_{m_{0}}$ is the original polarization when the molecules are built and $B_{c}$ corresponds to an effective magnetic field due to the different rotational states of the molecules and their occupation numbers. E.g., for ortho-hydrogen at temperatures below $200 \mathrm{~K}$ most molecules will be in the $J=1$ state and $B_{c}=5.4 \mathrm{mT}$. At $300 \mathrm{~K}$ about $12.5 \%$ of the molecules are in the $J=3$ state and $B_{c}$ is increased to $6.5 \mathrm{mT}$.

The distribution of the amount of particles with $n$ wall collisions in an ensemble of molecules in a storage cell is an exponential function:

$$
W(n)=1 / \alpha e^{-\alpha n} \quad\left(\int W(n) d n=1\right)
$$

Therefore, the average amount of wall collisions is $\bar{n}=\ln 2 / \alpha$ and depends on the geometry of the storage cell and the molecule-wall interaction. E.g., when elastic scattering of the molecules on the walls is assumed, the average amount of wall collisions can be calculated with Monte-Carlo simulations and is for a $400 \mathrm{~mm}$ long cell with an inner diameter of $10 \mathrm{~mm} \bar{n} \sim 125$.

With this weighting factor eq. 3.1 can be deconvolved to

$$
P_{m}(\bar{n}, B)=\frac{P_{m_{0}}}{1+\left(B_{c} / B\right)^{2}(\bar{n} / \ln 2)} .
$$

When the nuclear polarization of the molecules is now measured with the Lamb-shift polarimeter as a function of the magnetic field along the storage cell (see Fig. 2), the molecular polarization after the recombination process on a dedicated surface and the average amount of wall collisions can be deduced with a fit to eq. 3.3. In parallel, the polarization of the produced protons can be measured, when the Wien filter is used to separate them from the $\mathrm{H}_{2}^{+}$ions. In this case, one has to be aware that the protons can stem from ionized molecules or atoms inside the storage cell. Then, eq. 3.3 has to be modified to

$$
P_{p}(\bar{n}, B)=a P_{p}+b \frac{P_{m_{0}}}{1+\left(B_{c} / B\right)^{2}(\bar{n} / \ln 2)} .
$$

where $P_{p}$ is the nuclear polarization of the atoms in the storage cell, which can be assumed for high recombination to be close to the polarization of the atoms in the ABS beam itself. $a$ is the relative amount of protons produced from the atoms in the storage cell and $b$ is the relative amount of protons from the molecules $(a+b=1)$. Therefore, the measurement of the proton polarization allows the calculation of the recombination rate $c$. If one takes into account that the cross section for the production of protons from atoms is about 5.5 times higher, than for the production of protons from molecules follows:

$$
c:=\frac{2 b}{2 b+0.18 a}=\frac{1-a}{1-0.91 a}
$$

Fig. 2 shows a measurement of the polarization of the protons (red) and the $H_{2}^{+}$ions (green) as a function of the magnetic field inside the cell, when the cell surface was covered with Fomblin and was fed with hydrogen atoms in hyperfine state 3 . Due to a fit of eq. 3.3 on the $H_{2}^{+}$data (blue line) one can deduce $P_{m_{0}}=-0.84 \pm 0.02$ and $\bar{n}=225 \pm 41$. A fit of eq. 3.4 on the proton data (magenta) delivers $P_{m_{0}}=-0.81 \pm 0.02, \bar{n}=148 \pm 22$ and in combination with eq. $3.5 c=0.993 \pm 0.0054$. 


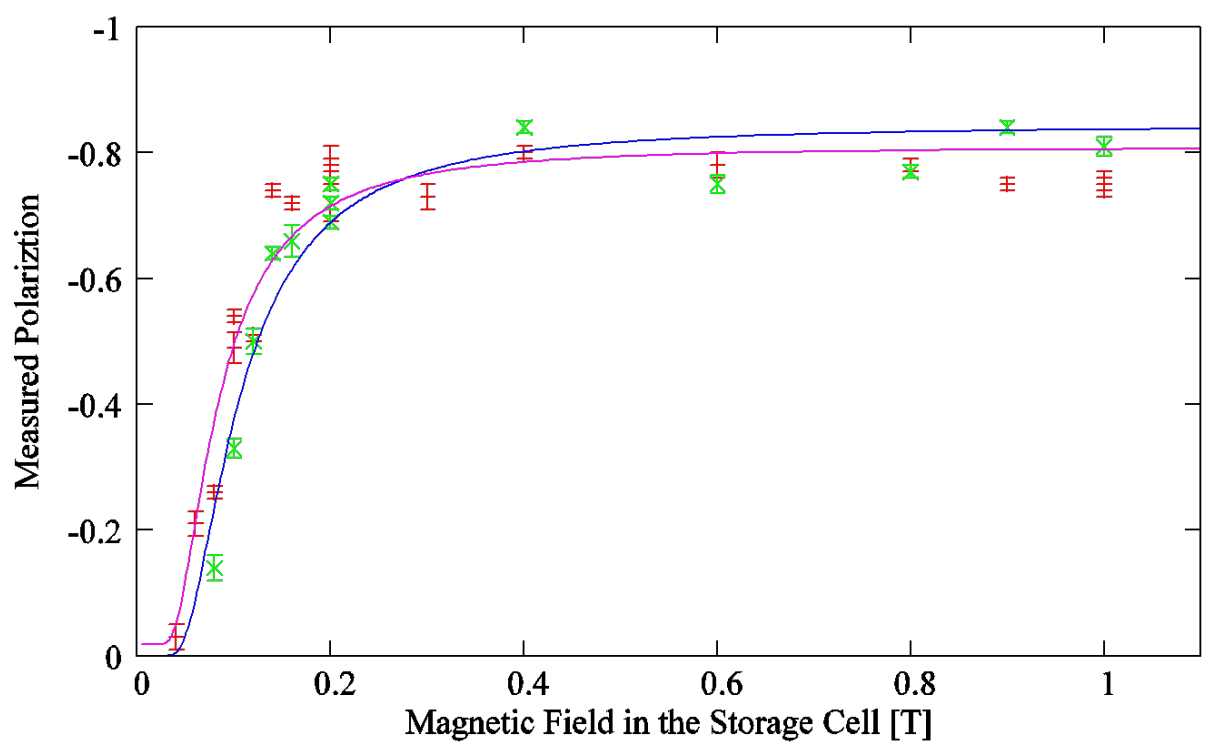

Figure 2: Measurement of the nuclear polarization of the $\mathrm{H}_{2}^{+}$ions (green) and protons (red) leaving a storage cell that was covered with Fomblin (Perfluoropolyether PFPE) and fed mainly with hydrogen atoms in hyperfine state 3 coming from the ABS. From a fit to the $H_{2}^{+}$data (blue) one get $P_{m_{0}}=-0.84 \pm 0.02$ and from the proton data $P_{m_{0}}=-0.81 \pm 0.02$ and a recombination rate $c=0.993 \pm 0.005$.

The maximum expected polarization due to the ABS beam quality was about $P_{m}=-0.87 \pm 0.02$. That the measured polarization for the $\mathrm{H}_{2}^{+}$ions is a little bit larger than for the protons can be explained with a possible background contribution of unpolarized protons because of the ionization of residual gases like $C_{n} H_{m}$ or from water on the surface, which will not deliver unpolarized $\mathrm{H}_{2}^{+}$ ions. The larger amount of wall collisions deduced from the $\mathrm{H}_{2}^{+}$ions can be explained by the much shorter average free path length of the $\mathrm{H}_{2}^{+}$ions inside the storage cell compared to the protons. I.e., when a proton is produced in the middle of the cell, where the average amount of wall collisions is rather small, it has a much larger chance to reach the Lamb-shift polarimeter than a $\mathrm{H}_{2}^{+}$ion. Therefore, most of the registered $\mathrm{H}_{2}^{+}$ions are produced at the end of the cell, where the average amount of wall collisions is higher.

\section{Conclusion}

With this apparatus it is now possible to measure in parallel the nuclear polarization of hydrogen (deuterium) atoms and molecules inside a storage cell and the recombination rate on different surfaces and temperatures. Thus, the nuclear spin is observable during the interactions of the atoms with the wall surface and the recombination into molecules. This allows a deeper view into chemical processes and the recombination process itself. E.g. it is obvious that a Fomblin surface at 100 $\mathrm{K}$ is able to change the electron spin of the hydrogen atom, but it does not change the nuclear spin. One possible explanation is the storage of the polarized proton on the Fomblin surface in potentials traps independently of the electron. A water surface seems to be unable to rotate the electron spin and, therefore, the recombination rate of atoms in a defined hyperfine state is small. If atoms with 
different electron spins are produced by the ABS, the recombination rate is increased immediately. In addition, it is possible to determine the amount of wall collisions of the molecules inside the cell. In combination with Monte-Carlo simulations the different kinds of molecule-wall interactions can be deduced.

To increase the figure of merit for experiments with polarized targets a cooled Fomblin surface can help in different ways:

- Molecules at the same temperature are slower and need more time to leave the storage cell.

- It is obvious that the cells can be cooled further than before, which again slows down the molecules.

- Because of the higher amount of wall collisions of the molecules in such a cell the target density is increased further.

The combination of all three effects can increase the target density by a factor of 3 without significant polarization losses.

For ortho-hydrogen molecules the rotational state $J=1$ is the ground state. Therefore, the molecules with parallel spin cannot be stored for a long time. But for ortho-deuterium, i.e. when both nuclear spins are parallel, the $J=0$ state is fully occupied at low temperatures. This should allow to freeze out the polarized deuterium molecules into ice, where the lifetime of the polarization should be very large. Collecting the polarized deuterium atoms, produced by an ABS for one day, might allow to get enough polarized fuel to feed a tokomak for one second. In this case, the lifetime of nuclear polarization in a fusion plasma can be determined and the different option for an increased energy output by "polarized fusion" can be investigated [11].

\section{Acknowledgment}

This work was supported by the International Science and Technology Center (ISTC No. 1861) and the Deutsche Forschungsgemeinschaft (DFG Project 436 RUS 113/977/0-1).

\section{References}

[1] J.F.J. van den Brand et al., Phys. Rev. Lett. 78 (1997) 1235.

[2] T. Wise et al., Phys. Rev. Lett. 87 (2001) 042701.

[3] P. Lenisa et al., Eur. Phys. J. D 29 (2004) 21.

[4] E. Steffens and W. Haeberli, Rep. Prog. Phys. 66, (2003) 1887.

[5] J.S. Price and W. Haeberli, Nucl. Instrum. Methods Phys. Res., Sect. A 349, (1994) 321.

[6] A. Airapetian et al., Nucl. Instrum. Methods Phys. Res., Sect. A 540, (2005) 68.

[7] J.S. Price and W. Haeberli, Nucl. Instr. Meth. A 326 (1993) 416.

[8] R. Engels et al., Rev. Sci. Instr. 74 (2003) 4607.

[9] M. Mikirtytchiants et al., Nucl. Instrum. Methods Phys. Res., Sect A721 (2013) 83.

[10] R. Engels et al., Rev. Sci. Instrum. 85 (2014) 103505.

[11] R. Engels et al.; Phys. Part. Nuc. 45 (2014) 341. 